Polarization interferometers: applications in microscopy and macroscopy. By $M$. Françon and S. MaLlick. Pp. 159. London: Wiley, 1971. Price $£ 4 \cdot 50$.

Polarization interferometers are ones in which the two coherent beams required for interference are produced from a single source by means of a birefringent beam-splitter, i.e. use is made of the separation of the $E$ and $O$ rays. The two resulting beams are necessarily poiarized at right angles, so there must be some arrangement for making their planes of polarization parallel again before interference can take place. There are many combinations of ways of carrying out these functions, and polarization interferometers can be employed for all the usual applications of interferometers. The field is therefore large and complicated, and the present monograph by Françon and Mallick aims to survey the possibilities and to bring together information otherwise only available from scattered sources. Their stated aim is to minimize the mathematics, and to concentrate on physical principles and on help with the actual use of the instruments. There are thus chapters on polarzation in general, and on birefringent beam-splitters and other components, followed by several chapters on typical applications, such as the observation of phase objects, the measurement of optical thickness, the testing of optical systems, and on polarization methods in interference microscopy. The authors are well qualified to survey this field as they are from the Institute $\mathrm{d}^{\prime} \mathrm{Optique}$ in Paris, where much of the work has been done, and they themselves have originated many of the instruments and variants described.

However, having said all this, the book itself is a little disappointing; the approach seems to be academic rather than practical, and the result is a textbook rather than a handbook. We are promised practical advice on how to set up the instruments, and in a few cases some useful component parameters are given: however, there is usually no such information, and the book would be much more helpful if we could have the benefit of the authors' considerable experience. Also, the diagrams of the instruments are conventionally schematic rather than practical, and in most cases give little idea as to what one should actually do. It would be useful to have more illustrations of the appearance of the fringes obtained; there are, in all, seven photographs of interference effects and about the same number of schematic diagrams of them, but usually there is nothing against which to confirm ones observations, or to assist in correcting maladjustments. A possibly more serious omission is that there is little indication of the advantage of using interferometers involving polarization, to be set against the fact that polarization components such as Savart plates and Wollaston prisms are very expensive. It is mentioned briefly in the introduction that polarization interferometers are simple to use, and that they are stable because the beam separation is small; two cases are described (differential interference microscopy and coherence measurement) in which a small separation is actually required and can conveniently be obtained by polarization. In the wide-angle Michelson interferometer a large entrance cone is achieved by making use of the reduced change of path difference with angle when a polarizing beam-splitter is used; however, one has to search for these cases in which some advantage is claimed, and even in these the results can be achieved by other means. It becomes evident from the many examples given that one can make polarization versions of interferometers to cover any of the usual applications - but it remains far from clear why one should do so, how much advantage is to be gained, and whether there are things that cannot be done any other way.

As regards completeness, one is of course bound to be able to find omissions from any compilation of this sort, but there are nevertheless a few rather surprising ones. For example, the Savart polariscope is described, and some variants of it, but there is no mention of the considerable improvements in sensitivity achieved - in Paris - in the version devised by Lyot and later improved by Dollfus. There is no mention of Sinton's polarization interferometer, or of the polarizationinterferencespectrometerdevised by Mertz, which is surely original enough to merit inclusion.

As stated in the introduction, theory was kept to a minimum in the body of the book, and it was a good idea to put some of the detailed theoretical discussions in appendices at the end. A minor fault here is probably a matter of editing - a formula used on p. 19 is said to be derived in Appendix l, but the resulting expression is given there in a slightly different form, giving the reader the exercise of showing it to be equi- valent; furthermore, a different symbol is used for the important angle it contains, which might cause some confusion.

On the whole, this book is useful in that it collects together information about some ingenious optical instruments which are probably not well enough known, and it may thereby encourage the reader to investigate further their undoubtedly useful properties.

Physics Department

J. E. GEAKE

UMIST

Manchester M60 1OD

England

\section{Principles and Practice of} $X$-ray Spectrometric Analysis. By Eugene P. Bertin. Pp. xxiv +679. New York: Plenum Press, 1970. Price $£ 17.50$, DM 150.00 .

The content of this book is an important attempt to fulfil the aims defined in the title. According to its author it is primarily intended for students and technicians either having no practical experience of $X$-ray spectrometric analysis or having some practical experience of the method but not enough formal instruction in its principles.

It is evident that sound basic knowledge of $X$-ray physics in general and spectroscopy in particular is implicitly involved throughout this text-book. The first two chapters (about one tenth of the whole) are explicitly devoted to a summary of the laws; the point of view is kept elementary, with little up-to-date theoretical justification. However, it should encourage the reader to take an interest in the fundamental aspects of the subject and to undertake more academic reading. It is mainly intended as a text-book for users of commercially available $X$-ray fluorescence spectrometers; as such, it will offer them a very valuable tool. Accordingly chapter 3 and the beginning of chapter 4 deal with $X$-ray secondary excitation. One also finds information on essential pieces of equipment (X-ray tubes, collimators, flat and curved crystal spectrometers, analyser crystals and multilayer films, various detectors, etc.), and how to use them appropriately. Non-dispersive analysis and (briefly in the last chapter) electron-probe microanalysis are also mentioned.

Four chapters of practical importance are dedicated to qualitative and semi- 